\title{
Nonuniform Self-Organized Dynamical States in Superconductors with Periodic Pinning
}

\author{
Vyacheslav R. Misko, ${ }^{1,2,3}$ Sergey Savel'ev, ${ }^{1,4}$ Alexander L. Rakhmanov, ${ }^{1,5}$ and Franco Nori ${ }^{1,2}$ \\ ${ }^{1}$ Frontier Research System, The Institute of Physical and Chemical Research (RIKEN), Wako-shi, Saitama, 351-0198, Japan \\ ${ }^{2}$ MCTP, Department of Physics, University of Michigan, Ann Arbor, Michigan 48109-1040, USA \\ ${ }^{3}$ Department of Physics, University of Antwerpen (CGB), B-2020 Antwerpen, Belgium \\ ${ }^{4}$ Department of Physics, Loughborough University, Loughborough LE11 3TU, United Kingdom \\ ${ }^{5}$ Institute for Theoretical and Applied Electrodynamics, Russian Academy of Sciences, 125412 Moscow, Russia
}

(Received 26 June 2005; published 29 March 2006)

\begin{abstract}
We consider magnetic flux moving in superconductors with periodic pinning arrays. We show that sample heating by moving vortices produces negative differential resistivity (NDR) of both $N$ and $S$ type (i.e., $N$ - and $S$-shaped) in the voltage-current characteristic (VI curve). The uniform flux flow state is unstable in the NDR region of the VI curve. Domain structures appear during the NDR part of the VI curve of an $N$ type, while a filamentary instability is observed for the NDR of an $S$ type. The simultaneous existence of the NDR of both types gives rise to the appearance of striking self-organized (both stationary and nonstationary) two-dimensional dynamical structures.
\end{abstract}

PACS numbers: 74.25.Qt

Semiconductor devices [1] exhibiting negative differential resistivity (NDR) and conductivity (NDC) have played a very important role in science and technology. These useful devices include [1]: Gunn effect diodes, $p$ - $n$ - $p$ - $n$-junctions, etc. Here we study superconducting analogs of these semiconductor devices. Table I briefly compares NDR in superconductors, semiconductors, plasmas, and manganites. Conceptually, the nonuniform selforganized structures (e.g., filaments and overheated domains with higher or lower electric fields) are related for superconductors, plasmas, and semiconductors. However, the physical mechanism giving rise to the instability of the homogeneous state can be different in each case.

The magnetic flux behavior in superconductors with artificial pinning sites has attracted considerable attention due to the possibility of constructing samples with desired properties [9-13]. Among such systems, samples with periodic arrays of pinning sites (PAPS) are studied intensely because advanced fabrication techniques allow to design well-defined periodic structures with controlled microscopic pinning parameters. For such systems, Ref. [14] has revealed the existence of several dynamical vortex phases, which are similar to the ones shown in the inset of Fig. 1. At low current density $j$, the vortices are pinned and their average velocity, $\bar{v}$, is zero (phase I). Here $\bar{v}=N_{v}^{-1} \sum_{i} \mathbf{v}_{i} \cdot \mathbf{x}$ is the average vortex velocity in the $x$ direction. At higher $j$, interstitial vortices start to move and the flux velocity increases with the drive (phase II). Then, a sharp jump in $\bar{v}(j)$ occurs since a fraction of the vortices depins and a very disordered uniform phase arises (phase III). This phase is analogous to the uniform electron motion in semiconductor devices. After increasing the applied current $j$ (for superconductors) or the applied voltage (for semiconductors), the vortex (electron) velocity shows a remarkable and nonintuitive sudden drop (i.e., the velocity drops even though the applied force increases). Indeed, when $j$ exceeds a threshold value, only incommen- surate vortex rows move (phase IV). Finally, the increasing driving force completely overcomes the pinning (phase V). The dependence $\bar{v}(j)$ describes the voltage-current characteristic (VI curve) since the electric field $E$ is related to $\bar{v}$ by $E=-\bar{v}(j) B / c$. Here we prove that the flux motion in samples with PAPS results in an unusual, for superconductors, $V I$ curve with NDR of the so-called $S$ type $[1,3]$. That is, within some interval of voltages there exist three different current values corresponding to a single electric field. Such types of $S$-shaped VI curves play a very important role in plasmas and semiconductors, and give rise to a filamentary instability when a uniform current flow breaks into filaments with lower and higher current densities [1].

Sufficiently strong disorder in the pinning array gives rise to the disappearance of the described dynamical phase transitions [14] and, consequently, the vanishing of the NDR for $S$-shaped $V I$ curves. Higher thermal fluctuations also result in such an effect. Experimentally, the current density, $j$, at which the flux flow regime is observed in superconductors, is usually high $[2,15]$, and the Joule heat should also affect the picture described above. An increase in temperature $T$ results in a decrease of the pinning force. Thus, the current density can decay with increasing electric field, and a $N$-shaped $V I$ curve with a NDR of $N$ type (red dashed line in Fig. 1) is commonly observed in superconductors for high current density [2,16]. The uniform state in samples with NDR of $N$ type is also unstable [1]; and a propagating resistive state boundary or the formation of resistive domains can destroy the uniform superconducting state $[2,4,16]$. For certain pinning parameters and cooling conditions, one can achieve a situation where the NDR of both $N$ and $S$ type simultaneously coexist in the $V I$ curve (Fig. 1). In this case, we predict remarkable flux flow instabilities.

Here, we study the effect of Joule heating and disorder on the VI curve of superconductors with PAPS. Based on analytical and numerical analysis of the VI curve, we find 
TABLE I. Comparison between nonuniform nonequilibrium states in superconductors, semiconductors, plasmas, and manganites with VI curves having negative differential resistivity (NDR). Since $I V$ curves in semiconductors map to $V I$ curves in superconductors, then NDC (for semiconductors) maps into NDR for superconductors. Here, $N(S)$-type shapes for semiconductors correspond to $S(N)$ type for superconductors [2]. The negative differential conductivity (NDC) found in [3] is analogous to the Gunn effect in semiconductors, where electron-charge modulations lead to steps in $j(E)$ in the NDC regime.

\begin{tabular}{|c|c|c|c|c|}
\hline & Superconductors & Semiconductors & Plasmas & Manganites \\
\hline Carriers & flux quanta & $\begin{array}{l}\text { charge quanta: } \\
\text { electrons or holes }\end{array}$ & electrons & electrons, holes \\
\hline $\begin{array}{l}\text { Characteristic } \\
\text { curve }\end{array}$ & $\begin{array}{l}\text { voltage-current } \\
\qquad(V I) \text { curve }\end{array}$ & $\begin{array}{l}\text { current-voltage } \\
(I V) \text { curve }\end{array}$ & $I V$ curve & $I V$ curve \\
\hline $\begin{array}{l}\text { Homogeneous } \\
\text { state }\end{array}$ & $\begin{array}{l}\text { homogeneous flux } \\
\text { and current flows }\end{array}$ & $\begin{array}{l}\text { homogeneous } \\
\text { current flow }\end{array}$ & $\begin{array}{l}\text { homogeneous } \\
\text { current flow }\end{array}$ & $\begin{array}{l}\text { homogeneous } \\
\text { current flow }\end{array}$ \\
\hline $\begin{array}{l}\text { Origin of } \\
S \text {-shape NDR }\end{array}$ & $\begin{array}{l}\text { incommensurate or commensurate } \\
\text { vortex dynamical phases in PAPS }\end{array}$ & $\begin{array}{l}\text { nonlinear electron } \\
\text { transport }\end{array}$ & ionization & $\cdots$ \\
\hline $\begin{array}{l}\text { Origin of } \\
N \text {-shape NDR }\end{array}$ & $\begin{array}{l}\text { overheating, Cooper pair } \\
\text { tunneling [4]; vortex-core: } \\
\text { shrinkage [5] }\left(T \approx T_{c}\right) \\
\text { expansion [3], driven [6] }\left(T \ll T_{c}\right)\end{array}$ & $\begin{array}{l}\text { overheating, electron, } \\
\text { or hole tunneling }\end{array}$ & heating [7] & heating [8] \\
\hline Filaments & supercurrent filaments & $\begin{array}{l}\text { normal current } \\
\text { filaments }\end{array}$ & pinch-effect & $\cdots$ \\
\hline Domains & $\begin{array}{l}\text { vortex-induced higher } E \\
\text { overheated domains }\end{array}$ & $\begin{array}{c}\text { higher } E \\
\text { overheated domains }\end{array}$ & $\begin{array}{c}\text { higher } E \text { overheated } \\
\text { domains }\end{array}$ & $\begin{array}{c}\text { higher } E \text { overheated } \\
\text { domains }\end{array}$ \\
\hline
\end{tabular}

the conditions under which the $V I$ curve has a NDR region of either $S$ type or $N$ type, or both. We discuss the effect of the shape of the NDR on the vortex and current flow. We argue that the coexistence of the NDR of both, $S$ and $N$ types, gives rise to the macroscopically nonuniform self-organized dynamical structures in the flux flow regime.

We numerically integrate the two-dimensional overdamped equations of motion $[14,17,18]$ for the flux lines driven, by the current $j$, in the $x$ direction over a square PAPS with lattice constant $a: \eta \mathbf{v}_{i}=\mathbf{F}_{i}^{v v}+\mathbf{F}_{i}^{v p}+\mathbf{F}_{i}^{T}+$ $\mathbf{F}_{d}$. Here $\eta$ is the flux flow viscosity [15], $\mathbf{v}_{i}$ is the velocity of $i$ th vortex, $\mathbf{F}_{i}^{v v}$ is the force acting on the $i$ th vortex per unit length due to the interaction with other vortices, $\mathbf{F}_{i}^{v p}$ is the $i$ th vortex-pin interaction, $F_{d}=j \phi_{0} / c$ is the driving force, and $\phi_{0}$ is the flux quantum. The thermal fluctuation contribution to the force, $\mathbf{F}_{i}^{T}$, satisfies: $\left\langle F_{i}^{T}(t)\right\rangle_{t}=0$ and $\left\langle F_{i}^{T}(t) F_{j}^{T}\left(t^{\prime}\right)\right\rangle_{t}=2 \eta k_{B} T \delta_{i j} \delta\left(t-t^{\prime}\right)$.

The vortex-vortex interaction is modeled by $\mathbf{F}_{i}^{v v}=$ $\left[\phi_{0}^{2} / 8 \pi^{2} \lambda^{3}(T)\right] \sum_{j=1}^{N_{v}} K_{1}\left(\left|\mathbf{r}_{i}-\mathbf{r}_{j}\right| / \lambda(T)\right) \hat{\mathbf{r}}_{i j}, K_{1}$ is the modified Bessel function, the summation is performed over the positions $\mathbf{r}_{j}$ of $N_{v}$ vortices in the sample, and $\hat{\mathbf{r}}_{i j}=\left(\mathbf{r}_{i}-\right.$ $\left.\mathbf{r}_{j}\right) /\left|\mathbf{r}_{i}-\mathbf{r}_{j}\right|$. The temperature dependence of the penetration depth $\lambda$ is approximated as $\lambda(T)=\lambda_{0}\left(1-T^{2} / T_{c}^{2}\right)^{-1 / 2}$. The Ginzburg-Landau formula for $H_{c 2}(T)$ is used and we assume that the ratio $\kappa_{\mathrm{GL}}=\lambda / \xi$ is temperature independent (here $\xi$ is the coherence length). The pinning is modeled as $N_{p}$ parabolic wells located at positions $\mathbf{r}_{k}^{(p)}$. The pinning force per unit length is $\mathbf{F}_{i}^{v p}=\left(F_{p}(T) / r_{p}\right) \times$ $\sum_{k=1}^{N_{p}}\left|\mathbf{r}_{i}-\mathbf{r}_{k}^{(p)}\right| \Theta\left(r_{p}-\left|\mathbf{r}_{i}-\mathbf{r}_{k}^{(p)}\right| / \lambda_{0}\right) \hat{\mathbf{r}}_{i k}^{(p)}$, where $r_{p}$ is the range of the pinning potential, $\Theta$ is the Heaviside step function, and $\hat{\mathbf{r}}_{i k}^{(p)}=\left(\mathbf{r}_{i}-\mathbf{r}_{k}^{(p)}\right) /\left|\mathbf{r}_{i}-\mathbf{r}_{k}^{(p)}\right|$. We estimate the maximum pinning force, $F_{p}=F_{p}(T)$, as $H_{c}^{2} \xi^{2} / r_{p}$ and, thus, $F_{p}(T)=F_{p 0}\left(1-T^{2} / T_{c}^{2}\right)$.

For brevity, the simulations shown here are for $18 \times 12$ $\lambda_{0}^{2}$ periodic cells and at magnetic fields near the first matching field, $B_{\phi}=\phi_{0} / a^{2}$, where $N_{p}=N_{v}$. First, we start with a high-temperature vortex liquid. Then, the temperature is slowly decreased down to $T=0$. When cooling down, vortices adjust themselves to minimize their energy, simulating field-cooled experiments. Then we in-

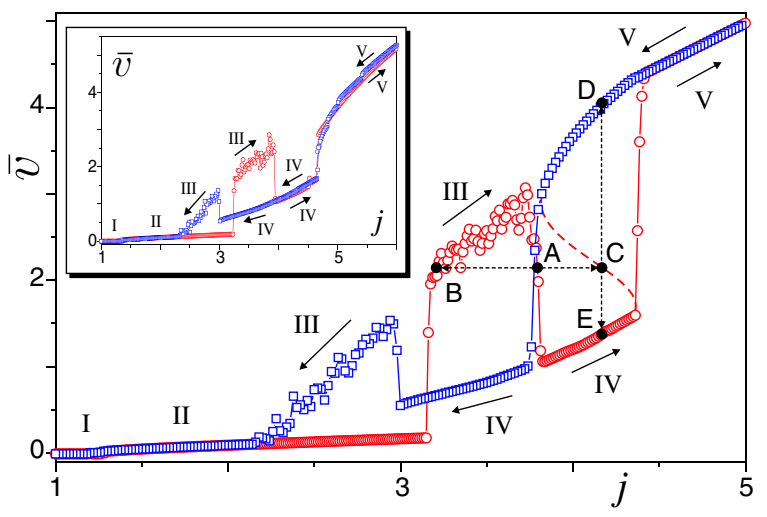

FIG. 1 (color online). The average vortex velocity $\bar{v} \propto E$ vs current $j$ for $B / B_{\Phi}=1.074, r_{p}=0.2 \lambda_{0}$, and $F_{p} / F_{0}=2$ for increasing (red open circles) and for decreasing (blue open squares) $j$. State $A$ is unstable and the sample divides into filaments, some in states $B$ and some in $C$. State $C$ is also unstable. The corresponding stable states are on the lower (point $E$ ) and on the upper (point $D$ ) $V I$-curve branches. Inset: $V I$ curve when no heating effects are taken into account. 
crease the driving current and compute the average vortex velocity $\bar{v}(j)$.

The average power of Joule heating per unit volume is $j E=j \bar{v} B / c$. We assume that the temperature relaxation length is larger than any local scale and the sample thickness. Under such conditions, the local temperature increase due to vortex motion can be found from the heat balance equation [2]: $h_{0} S\left(T-T_{0}\right)=\bar{v} j B V / c$, where $h_{0}$ is the heat transfer coefficient, $T_{0}$ the ambient temperature, and $S$ and $V$ are the sample surface and volume. Further, we shall assume that $T_{0} \ll T_{c}$ and neglect $T_{0}$. We define the dimensionless driving force as $f_{d}=j / j_{0}$ and introduce the dimensionless parameters $V_{x}=\bar{v} / v_{0}$ and $b=B / B_{\phi}$, where $v_{0}=c^{2} / 4 \pi \kappa_{\mathrm{GL}}^{2} \sigma_{n} \lambda_{0}$, and $j_{0}=c \phi_{0} / 8 \pi^{2} \lambda_{0}^{3}$. We assume that the normal state conductivity $\sigma_{n}$ is temperature independent. As a result, the temperature and the driving force are related by: $T / T_{c}=K_{\mathrm{th}} V_{x} f_{d} b$, where $K_{\mathrm{th}}=$ $j_{0} v_{0} B_{\phi} V / c h_{0} T_{c} S$ is the ratio of the characteristic heat release to heat removal. Using the rough estimates $\lambda_{0}=$ $2000 \AA, \quad \kappa_{\mathrm{GL}}=100, \quad \sigma_{n}=10^{16} \mathrm{~s}^{-1}, \quad V / S=1000 \AA$, $T_{c}=90 \mathrm{~K}, B_{\phi}=500 \mathrm{G}$, and $h_{0}=1 \mathrm{~W} / \mathrm{cm}^{2} \mathrm{~K}$, we find $K_{\text {th }}=0.05-0.06$ and $F_{p 0}$ is of the order of $F_{0}=\phi_{0} j_{0} / c$. In the simulations, we used $K_{\text {th }}=0.0525$.

The velocity $\bar{v}$ versus current $j$ (which coincides with the sample $V I$ curve in dimensionless variables) is presented in Fig. 1 for increasing and for decreasing $j$ [if we neglect the heating effect, $\bar{v}(j)$ has the shape shown in the inset of Fig. 1, similar to Ref. [14] ]. For low currents, $j \lesssim$ 3 , the effect of heating is negligible; for $j \gtrsim 3.7$, the behavior of $\bar{v}(j)$ drastically changes, compared to the nonheating case shown in the inset of Fig. 1. In particular, an abrupt transition occurs between regimes IV and V. The most pronounced feature related to heating is the appearance of hysteresis in regions IV and V: the overheated vortex lattice (for decreasing $j$ ) keeps moving as a whole at lower currents than the "cold" one (for increasing $j$ ). As a result, we obtain a complex $N$ - and $S$-type $V I$ curve, characterized by two kinds of instabilities. For example, if the current density exceeds the value $j \approx 3.7$ (point $A$ ), the uniform current flow is unstable and the so-called filamentary instability [1] occurs. Consequently, the current flow breaks into supercurrent filaments, some with lower $j_{B}$ (state B) and others with higher $j_{C}$ (state C). The state C is, in its turn, unstable. The corresponding stable states are on the lower (E) and on the upper (D) VI-curve branches.

Note that a small amount of pinning disorder influences $\bar{v}(j)$ and can lead to the disappearance of phase III [19]. However, the robust hysteresis, related to heating, remains.

For a sample included in an electric circuit (see inset in Fig. 2), the circuit equation is $L \dot{I}+R I+l E=U$, where $I=j A, L$ and $R$ are the inductance and resistance, $A$ and $l$ are the sample cross section and length, and $U$ is a constant voltage. The circuit and Maxwell equations describe the development of small field perturbations $\delta \mathbf{E}$ and $\delta \mathbf{B}$ and current $\delta j$. We seek perturbations of the form $\delta E, \delta B, \delta j \propto$ $\exp \left(\nu t / t_{0}\right)$, where $\nu$ is the value to be found and $t_{0}=L / R$.

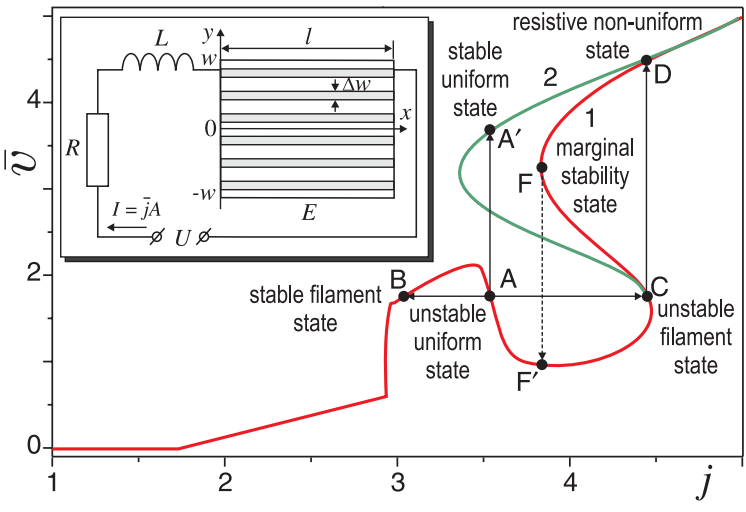

FIG. 2 (color online). Schematic VI curve of the superconductor for two different values of the hysteresis due to overheating. The more pronounced hysteresis loop (shown by the green solid line) corresponds to a larger value of the characteristic heat release. Inset: electrical circuit and the sample with filaments.

An instability develops if $\operatorname{Re}(\nu)>0$. In general, we should consider also the thermal equation, but for filamentary instabilities the temperature rise is not of importance. To find the instability criterion we consider $y$-dependent perturbations [1]. In such a geometry, $\delta \mathbf{E}$ has only the $x$ component, while $\delta \mathbf{B}$ has only the $z$ component. Using $\delta B=c t_{0} \delta E^{\prime} / \nu w$, we find from the circuit and Maxwell equations that:

$$
\begin{gathered}
(\nu+1) \delta \bar{j}+\rho_{c}^{-1} \delta \bar{E}=0, \\
\delta E^{\prime \prime}-\beta \delta E^{\prime}-\frac{\nu t_{s}}{t_{0}} \delta E=0,
\end{gathered}
$$

where $\delta \bar{j}$ and $\delta \bar{E}$ are the values averaged over the cross section, prime is differentiation over the dimensionless coordinate $y / w, w$ is the sample half-width, $\rho_{c}=R A / l$, $t_{s}=\left(4 \pi w^{2} / c^{2}\right)(\partial j / \partial E), \quad \beta=(4 \pi w / c)(\partial j / \partial B)$ are parameters, which are either positive or negative depending on the part of the $V I$ curve for given background fields $E$ and $B$. All quantities are averaged over a volume which includes a large number of vortices.

The first boundary condition to Eq. (2), $\delta B(w)=$ $-\delta B(-w)$, is obtained assuming that the applied magnetic field $B$ is constant. From Maxwell equations we derive $\delta B(w)-\delta B(-w)=8 \pi w \delta \bar{j} / c$. Using these $\delta B$ 's, and substituting $\delta \bar{j}$ from Eq. (1), we obtain the second boundary condition $\delta E^{\prime}(w)=-\gamma \nu \delta \bar{E} /(\nu+1)$, where $\gamma=$ $4 \pi w^{2} l / c^{2} A L$. Substituting the solution of Eq. (2), $\delta E=$ $C_{1} \exp \left(p_{1} y / w\right)+C_{2} \exp \left(p_{2} y / w\right)$, to the boundary conditions and requiring a zero determinant for the obtained set of linear equations for constants $C_{i}$, we find the equation for the eigenvalues $\nu$

$$
\left[p_{2}+\frac{\gamma \nu}{(\nu+1) p_{2}}\right] \frac{p_{1} \tanh p_{2}}{p_{2} \tanh p_{1}}=p_{1}+\frac{\gamma \nu}{(\nu+1) p_{1}},
$$

where $p_{1,2}=\beta / 2 \pm \sqrt{\beta^{2} / 4+\nu t_{s} / t_{0}}$.

For small values of $|\partial j / \partial B|$, the solution of Eq. (3) can be found explicitly (with accuracy up to $\left|\beta^{2}\right|$ ): $\nu=-1-$ $\gamma t_{0} / t_{s}$. It follows from this relation that the instability 
occurs only at the $V I$-curve branch with NDR, if the drop of the voltage is large: $|\partial E / \partial j|>\rho_{c}$. The characteristic size of the arising filamentary structure is of the order of $\Delta w \propto w /\left|p_{1}\right|=w / \sqrt{\left|\gamma+t_{s} / t_{0}\right|}$. If $\left|\gamma t_{s} / t_{0}\right| \gg 1$, the filament width is small, $\Delta w \ll w$. Thus, the sample with NDR in the $V I$ curve of $S$ type divides into small filaments with different current densities (in different dynamic flux flow phases III and IV) at $R l / A \ll|\partial E / \partial j|$ and $L \ll$ $4 \pi l / c^{2} A$. The obtained results are valid if $\rho_{c}^{-1}|\partial E / \partial j| \gg$ $[(4 \pi w / c) \partial j / \partial B]^{2}$. The instability occurs only if the lefthand side of this last inequality is higher than unity; the right-hand side is much smaller than 1 for the parameter range used above if $w<1 \mathrm{~mm}$. In the stationary inhomogeneous state that arises after development of the instability, the electric field should be uniform over the sample.

A more complex dynamics appears when the VI curve has simultaneously both NDR parts of $N$ and $S$ types (see Fig. 2). In this case, the filaments with higher current density $j_{C}$ are unstable if the system is far from the voltage-bias regime [1,2]. The instability of the filament with an $N$-type $V I$ curve results in the switch of the filament into the overheated state [16] $D$, giving rise to a nonuniform electric field distribution in the sample and, as a result, to nonzero $\dot{B}$. Thus, the state that appears after the instability develops is nonstationary. We consider two possible $V I$ curves (type 1 red, type 2 green) shown in Fig. 2. In state $D$ the flux lines move fast, which is accompanied with the acceleration of the flux flow in the lowercurrent filaments. In the nearly current-biased mode [1], if the $V I$ curve is of type 2, the sample comes to the uniform stable state $A^{\prime}$ with the current density $j_{A}$. However, if the $V I$ curve has the form shown in the (red) curve 1, the stable uniform state with the current density $j_{A}$ does not exist. In this case the high electric field state moves from point $D$ to point $F$ and falls down to a lower branch of the $V I$ curve (point $F^{\prime}$ ). In this state, the electric field is also nonuniform and the lower and higher resistivity states will move towards A. However, this state is unstable and the cycle of transitions will be repeated $\left(A \rightarrow C \rightarrow D \rightarrow F \rightarrow F^{\prime} \rightarrow\right.$ $A)$. Such a cyclical dynamic state could be realized in the form of flowing either stripes or resistive domain walls moving along the filaments.

It is important to stress that the described cyclical 5-step dynamics $\left(A \rightarrow C \rightarrow D \rightarrow F \rightarrow F^{\prime} \rightarrow A\right.$ ) obtained for the $I V$ curve having NDR of both $N$ and $S$ types cannot be realized for $I V$ curves with either only $N$ or only $S$ type of NDR. The appearance of the nonstationary oscillatory regime for stationary external conditions is very unusual. This cyclic dynamics could be extended to different physical systems, e.g., plasmas or superconductors without artificial pins driven by a current flowing along the externally applied magnetic field [20]. Moreover, the predicted dynamical behavior (which can be generalized for several other systems, e.g., semiconductors) is potentially useful for the transformation of a dc input into either an ac current or a voltage output which can be controlled by the parame- ters of the external circuit. In a broader sense, this general class of cyclic dynamics (ac output from dc input) is also found in other important nonlinear systems, like the dc Josephson effect.

In summary, for superconductors with periodic pinning arrays with certain pinning and heat transfer characteristics, we derive VI curves with NDR of $N$ type, $S$ type, or both. Complex dynamics and regimes including domain structures and filamentary instabilities appear when the $V I$ curve has both $S$ and $N$ types of NDR. We analyzed the self-organized nonuniform dynamical regimes for these instabilities.

This work was supported in part by ARDA and NSA under AFOSR Contract No. F49620-02-1-0334, and by the US NSF Grant No. EIA-0130383.

[1] See, e.g., E. Schöll, Nonequilibrium Phase Transitions in Semiconductors (Springer, New York, 1987); M. P. Shaw, V. V. Mitin, E. Schöll, and H.L. Grubin, The Physics of Instabilities in Solid State Electron Devices (Plenum, New York, 1992).

[2] A. V. Gurevich, R. G. Mints, and A.L. Rakhmanov, The Physics of Composite Superconductors (Begell, New York, 1997).

[3] M. N. Kunchur et al., Phys. Rev. Lett. 84, 5204 (2000); 87, 177001 (2001).

[4] A. Wehner et al., Phys. Rev. B 63, 144511 (2001); O. M. Stoll et al., Phys. Rev. Lett. 81, 2994 (1998).

[5] A. I. Larkin and Yu. N. Ovchinnikov, Zh. Eksp. Teor. Fiz. 68, 1915 (1975) [Sov. Phys. JETP 41, 960 (1976)].

[6] S. G. Doettinger et al., Phys. Rev. Lett. 73, 1691 (1994); A. V. Samoilov et al., ibid. 75, 4118 (1995); Z. L. Xiao et al., Phys. Rev. B 53, 15265 (1996).

[7] F. F. Cap, Handbook on Plasma Instabilities (Academic, New York, 1976).

[8] M. Tokunaga et al., Phys. Rev. Lett. 94, 157203 (2005).

[9] M. Baert et al., Phys. Rev. Lett. 74, 3269 (1995); V. V. Moshchalkov et al., Phys. Rev. B 54, 7385 (1996).

[10] J.E. Villegas et al., Science 302, 1188 (2003); Phys. Rev. B 68, 224504 (2003); M. I. Montero et al., Europhys. Lett. 63, 118 (2003).

[11] A. M. Castellanos et al., Appl. Phys. Lett. 71, 962 (1997); R. Wördenweber et al., Phys. Rev. B 69, 184504 (2004).

[12] L. Van Look et al., Phys. Rev. B 66, 214511 (2002).

[13] A. V. Silhanek et al., Phys. Rev. B 67, 064502 (2003).

[14] C. Reichhardt et al., Phys. Rev. Lett. 78, 2648 (1997); Phys. Rev. B 58, 6534 (1998).

[15] G. Blatter et al., Rev. Mod. Phys. 66, 1125 (1994); E. H. Brandt, Rep. Prog. Phys. 58, 1465 (1995).

[16] A. V. Gurevich et al., Rev. Mod. Phys. 59, 941 (1987).

[17] F. Nori, Science 271, 1373 (1996).

[18] B. Y. Zhu et al., Phys. Rev. B 68, 014514 (2003); Physica (Amsterdam) 18E, 318 (2003); Physica (Amsterdam) 388389C, 665 (2003); 404C, 260 (2004); Phys. Rev. Lett. 92 , 180602 (2004).

[19] V. R. Misko et al. (to be published).

[20] See, e.g., E. H. Brandt, J. Low Temp. Phys. 39, 41 (1980). 